\title{
Musashi-1 expression and clinicopathological significance in young gastric cancer patients: A matched case-control study
}

\author{
JI EUN CHOI ${ }^{1}$, JUN SANG BAE ${ }^{1}$, JU HYUNG LEE ${ }^{2}$, KYU YUN JANG $^{1}$, \\ MYOUNG JA CHUNG ${ }^{1}$ and WOO SUNG MOON ${ }^{1}$ \\ ${ }^{1}$ Department of Pathology, ${ }^{2}$ Preventive Medicine, Chonbuk National University, Medical School, \\ Research Institute of Clinical Medicine of Chonbuk National University Hospital and \\ Research Institute for Endocrine Sciences, Jeonju 561-756, Republic of Korea
}

Received November 18, 2013; Accepted December 29, 2013

DOI: $10.3892 /$ ijo.2014.2263

\begin{abstract}
Musashi-1 (Msi-1) is proposed to be a marker of progenitor cells in the human gastric mucosa. We examined Msi-1 expression and localization in surgical specimens of gastric cancer in young patients using immunohistochemistry and tested associations of Msi-1 expression with clinicopathological features. Patients $(n=611)$ with gastric cancer who underwent radical gastrectomy were included in the present study. To minimize confounding effects, we matched 26 gastric cancer patients 30 years of age or younger (age $\leq 30$ ) with 26 patients 60 years of age or older (age $\geq 60$ ). The groups were matched by gender, tumor histological type and tumor stage. Gastric cancer in the younger patients was significantly associated with female gender and with diffuse histological type, compared with 585 gastric cancer patients older than 31 years. Msi-1 expression was more frequently upregulated in gastric cancer in young patients than in patients older than 60 years. Msi-1 expression was significantly associated with diffuse histological type, depth of tumor invasion, lymph node metastasis and tumor stage in the 26 young patients with gastric cancer. Univariate Kaplan-Meier survival analysis identified Msi-1 expression as a significantly negative factor in the survival of young gastric cancer patients. However, Msi-1 expression was not significantly associated with survival in the 26 matched older patients. According to mucin phenotype, the gastric foveolar type predominated in Msi-1-positive gastric cancers. Our principal findings included a significantly higher level of Msi-1 expression in younger gastric cancer patients compared to older ones, and a probable association of tumor
\end{abstract}

Correspondence to: Professor Woo Sung Moon, Department of Pathology, Chonbuk National University Medical School, 567 Baekje-daero, Deokjin-gu, Jeonju-si, Jeollabuk-do 561-756, Republic of Korea

E-mail: mws@chonbuk.ac.kr

Key words: carcinoma, gastric, young adult
Msi-1 expression in young gastric cancer patients with more aggressive tumor type.

\section{Introduction}

Gastric cancer is one of the most common malignant tumors of the gastrointestinal system and cancer-related causes of death worldwide. Although screening has reduced the incidence of advanced disease, gastric cancer remains the most common malignancy in many countries including Korea $(1,2)$. The average age at diagnosis of gastric cancer is approximately 60 years; 2 to $9 \%$ of patients are younger than 40 years and most are older than 35 years. Patients younger than 30 years with gastric cancer are very rare $(3,4)$. Evidence suggests that gastric cancers occurring early in life are more aggressive and have a worse prognosis than those occurring later. Such a prognosis may be attributable to delay in diagnosis, intrinsically aggressive disease, and a higher frequency of undifferentiated tumor type in younger patients (4-7); however, these points remain controversial.

One current theory of cancer origin centers on cancer stem cells (CSCs) or cancer initiating cells, which are generally defined as a small population of cells within a tumor that possesses both the capacity for self-renewal and potential to generate multiple cell lineages (pluripotency) $(8,9)$. CSCs are hypothesized to participate in tumor initiation, invasion, metastasis and drug resistance. Newly discovered stem cell-associated genes have emerged as diagnostic and prognostic markers in many types of cancers (8-10). Musashi-1 (Msi-1) is a highly conserved neural RNA-binding protein, initially identified in Drosophlia where it is required for the early asymmetric division of sensory neural precursor cells (11). In stomach, stem cells present in the proliferative zone of the neck and isthmus region give rise to all of the differentiated epithelial cell types (12). Akasaka et al report Msi-1 expression predominantly in epithelial cells of the isthmus/neck region in human antrum, and Msi-1-positive cells in the proliferative regions do not co-express PCNA or Ki-67, suggesting that Msi-1 is a marker for cells with progenitor characteristics before active proliferation in the human stomach (13). Increasing evidence supports the activities of Msi-1 in cell proliferation, differentiation, inhibition 
of apoptosis and post-translational modification of proteins, suggesting potential roles for this protein in tumorigenesis $(14,15)$. However, the expression and clinical significance of Msi-1 in gastric cancer, and in particular, the relationship of Msi-1 expression to age at diagnosis of gastric cancer, present questions that are largely unexplored.

In the present study, we examined Msi-1 expression in surgical specimens of gastric cancers and analyzed relationships between Msi-1 expression and clinicopathologic features with respect to age (age $\leq 30$ versus age $\geq 60$ ).

\section{Materials and methods}

Patients. From 2001 through 2011, radical gastrectomies were performed in 2,757 patients at Chonbuk National University Hospital, Korea. Of these, 26 patients $(0.9 \%)$ were younger than 30 years. For this comparative study of gastric cancer between age groups, data for 585 patients older than 31 years who were treated by surgical resection between 2005 and 2007 were selected. Clinical and pathological information were collected from the hospital cancer registry database and from individual medical records, and the data for patients 30 years of age and younger $(n=26)$ were compared with data for patients 31 years of age and older $(n=585)$. To evaluate relationships between Msi-1 expression and clinicopathological data, and to compare these relationships between younger and older patients, we matched the 26 young patients with gastric cancer (age $\leq 30$ ) with 26 older patients (age $\geq 60$, mean age, 68.8 years; 11 male and 15 female). Matching was conducted for gender, tumor histologic type and tumor stage. The 2010 American Joint Committee on Cancer Staging TNM system was used for clinical and pathological staging (16). Gastric cancers were classified according to the WHO classification (17). The Institutional Review Board (IRB) of Chonbuk National University Hospital approved this study, and waived the requirement for written informed consent because of the retrospective design.

Immunohistochemical staining. A representative formalin-fixed, paraffin-embedded, 4- $\mu \mathrm{m}$ section was obtained from the gastric cancer of each patient. Immunohistochemical staining was performed using a polymer detection system with the Bond-Max Automatic stainer (Leica, Bannockburn, IL) in accordance with the manufacturer's instructions. Briefly, after antigen retrieval (microwave treatment for $10 \mathrm{~min}$ in $0.01 \mathrm{M}$ EDTA buffer, $\mathrm{pH} 9$ ), the slide was incubated with anti-Msi-1 antibody (Abcam, Cambridge, UK) for $30 \mathrm{~min}$. To determine the relationship between Msi-1 expression and proliferative activity in the tumor cells, we performed staining for $\mathrm{Ki}-67$ (Dako, Carpinteria, CA). In addition, we examined expression profiles of CD 10 (Cell Marque, Hot Springs, AK), Muc2 (Novocastra, Newcastle upon Tyne, UK), Muc5Ac (Novocastra), and Muc6 (Novocastra) to relate Msi-1 expression to mucin phenotype. CD10 and Muc2 are markers for the intestinal cells, and Muc5Ac and Muc6 are markers for gastric foveolar and gastric pyloric cells, respectively. Peroxidase activity was detected with the enzyme substrate 3-amino-9-ethyl carbazole. For negative controls, sections were treated in the same way except that they were incubated with citrate-buffered saline instead of the primary antibody.

\section{Immunohistochemical analysis}

Msi-1 expression in normal stomach. Areas with the highest densities of Msi-1 positive cells in non-malignant tissues were identified on slides by microscopic examination at $\mathrm{x} 4$ magnification. Msi-1-positive cells were counted in five areas under x400 magnification (high power field; HPF).

Msi-1 expression and mucin phenotype in gastric cancer. The immunostaining results, positive or negative, for Msi-1 or mucin phenotype were rated according to a score calculated by multiplying the area of the stain to the intensity of the stain. The area of staining was scored as follows: 0 (<5\%), 1 (6-30\%), 2 (31-60\%), and 3 ( $\geq 61 \%$ of tumor cells). The intensity of cell staining was scored categorically as follows: 0 , no immunostaining; 1 , weak; 2, moderate; and 3 , strong. The maximum combined score was 9 and the minimum score was 0 . If the product of area and intensity scores was equal to or higher than 3 , immunostaining was determined to be positive; otherwise, the tumor was determined to be negative. The cut-off score for positive Msi-1 expression was determined by receiver-operating characteristic (ROC) curve analysis. Samples with at least $10 \%$ of tumor cell nuclei stained for Ki-67 were determined to be positive.

Cell lines and culture. We also performed western blot analysis of Msi-1 expression in four different gastric cancer cell lines, MKN-28, MKN-45, NCI-N87 and KATO III (Korean Cell Line Bank, Seoul, Korea). All cell lines were cultured in RPMI-1640 medium supplemented with penicillin and streptomycin $(100 \mathrm{U} / \mathrm{ml})$ and $10 \%$ fetal bovine serum (Gibco-BRL, Gaithersburg, MD), at $37^{\circ} \mathrm{C}$ and with $5 \% \mathrm{CO}_{2}$ in a humidified incubator.

Western blot analysis. Cultured cells were extracted with PRO-PREP Protein Extraction Solution (Intron Biotechnology, Seoul, Korea). Briefly, proteins were resolved by SDS-PAGE on $12 \%$ gels and electrotransferred to polyvinylidene difluoride (PVDF) membranes using a semidry transfer method (Bio-Rad, Hercules, CA). The membrane was then blocked with 5\% non-fat dry milk in Tris-buffered saline (TBS)-0.1\% Tween-20 (15 mM NaCl, 100 mM Tris- $\mathrm{HCl}$, $\mathrm{pH} 7.5)$ for $1 \mathrm{~h}$. The membrane was incubated with anti-Msi-1 antibody (Abcam) overnight at $4^{\circ} \mathrm{C}$. Proteins on membranes were visualized using an enhanced chemiluminescence (ECL) detection system (Amersham Biosciences, Buckinghamshire, UK) and exposed to a luminescent image analyzer (LAS-3000, Fuji Film, Tokyo, Japan).

Statistical analysis. Data are expressed as the mean \pm SE. Comparisons between Msi-1 expression and clinicopathological characteristics were assessed using the $\chi^{2}$ test. The Mann-Whitney Rank Sum test was used to evaluate differences in Msi-1 expression between two groups. Survival analyses were performed using the Kaplan-Meier method, and differences in survival between different age groups were analyzed by the log-rank test. P-values $<0.05$ were considered to indicate statistically significant difference. 
Table I. Clinicopathological data for 611 patients with gastric cancer.

\begin{tabular}{lrrr}
\hline Characteristics & $\begin{array}{r}\text { Age } \leq 30 \\
\mathrm{n}=26(\%)\end{array}$ & $\begin{array}{c}\text { Age }>30 \\
\mathrm{n}=585(\%)\end{array}$ & $\mathrm{p}$-value \\
\hline Gender & & & 0.009 \\
$\quad$ Female & $15(57.7)$ & $193(33.0)$ & \\
$\quad$ Male & $11(42.3)$ & $392(67.0)$ & \\
Differentiation & & & 0.001 \\
$\quad$ Well & $0(0.0)$ & $100(17.1)$ & \\
$\quad$ Moderate & $4(15.4)$ & $215(36.7)$ & \\
Poorly & $11(42.3)$ & $148(25.3)$ & \\
Signet ring cell & $11(42.3)$ & $93(15.9)$ & \\
Others & $0(0.0)$ & $29(5.0)$ & \\
Lauren classification & & & $<0.001$ \\
Intestinal & $4(15.4)$ & $342(58.5)$ & \\
$\quad$ Diffuse & $22(84.6)$ & $243(41.5)$ & \\
T classification & & & 0.903 \\
T1, T2 & $16(61.5)$ & $353(60.3)$ & \\
$\quad$ T3, T4 & $10(38.5)$ & $232(39.7)$ & \\
Node metastasis & & & 0.977 \\
No & $19(73.1)$ & $429(73.3)$ & \\
$\quad$ Yes & $7(26.9)$ & $156(26.7)$ & \\
Overall stage & & & 0.814 \\
I & $16(61.5)$ & $386(66.0)$ & \\
II & $4(15.4)$ & $93(15.9)$ & \\
III & $6(23.1)$ & $106(18.1)$ & \\
\hline
\end{tabular}

\section{Results}

Clinicopathological data. Among the 611 patients with gastric cancer studied, 26 patients were younger than age 30 (mean age of patients, 27.8 years; 11 male and 15 female) and 585 were older than age 31 (mean age of patients, 60.5 years; 392 male and 193 female). Among tumors from the 26 younger patients, $4(15.4 \%)$ were moderately differentiated, $11(42.3 \%)$ were poorly differentiated and $11(42.3 \%)$ were signet ring cell carcinomas. According to the Lauren classification (18), 22 tumors (84.6\%) were of the diffuse type and $4(15.4 \%)$ were of intestinal type. Sixteen patients $(61.5 \%)$, were at stage I, four (15.4\%) were stage II, and six (23.1\%) were stage III. Among tumors from the 585 patients older than 31 years of age, $100(17.1 \%)$ were well-differentiated, $215(36.7 \%)$ were moderately differentiated, $148(25.3 \%)$ were poorly differentiated, $93(15.9 \%)$ were signet ring cell carcinoma and $29(5.0 \%)$ were of other types. Based on the Lauren classification, 243 (41.5\%) tumors were of a diffuse type and $342(58.5 \%)$ were of intestinal type. Numbers of patients at stages I, II and III were 386 (66.0\%), 93 (15.9\%) and $106(18.1 \%)$, respectively.

Clinicopathological features of younger patients with gastric cancer. Gastric cancers in the 26 patients age 30 or younger were significantly associated with female gender $(\mathrm{p}=0.009)$ and diffuse type cancer (poorly differentiated and signet ring

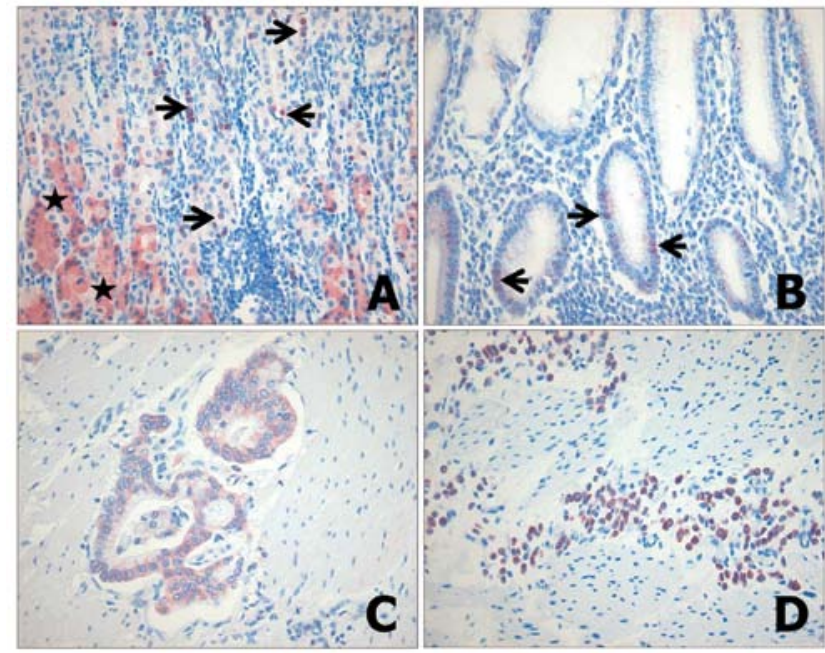

Figure 1. (A) Msi-1 expression was observed mainly in cell nuclei in the proliferating zone of fundic glands (arrows). Zymogenic cells showed nonspecific cytoplasmic immunoreactivity (stars). (B) Msi-1 expression was apparent in epithelial cells of the isthmus/neck region in the adult human antrum (arrows). (C) Msi-1 expression in moderately differentiated carcinoma cells. (D) Strong Msi-1 expression in poorly differentiated carcinoma cells infiltrating the muscular layer.

cell types, $\mathrm{p}=0.001$ ), as compared to gastric cancers in the 585 patients older than age 31. Clinicopathological features of the younger gastric cancer patients compared with the older patients are summarized in Table I.

Expression of Msi-1 in gastric cancer tissue and cells. Similar to a previous study (13), we observed Msi-1 expression predominantly in epithelial cells of the proliferative zone (isthmus/neck) in normal stomach, as revealed by nuclear and/or cytoplasmic immunoreactivity (Fig. 1A and B). In contrast, the surface foveolar cells and epithelial cells in the basal regions of the glands did not show Msi-1 immunoreactivity. Chief cells in the base of fundic mucosa showed weak, non-specific, and mainly cytoplasmic Msi-1 immunoreactivity. Only minimal or focal staining was present in gastric glands showing intestinal metaplasia in elderly patients, and this did not satisfy positive criteria. Non-tumor tissues from the younger patients showed significantly higher numbers of Msi-1 positive cells $(15.43 \pm 1.15 / \mathrm{HPF})$ than those of the older patients $(9.17 \pm 0.70 / \mathrm{HPF})(\mathrm{p}>0.001)$. In gastric tumor tissues, Msi-1 was detected predominantly in cytoplasm but also in nuclei of some tumor cells (Fig. 1C and D). Msi-1 expression was detected more frequently in tumors from the younger patients (26.9\%) than in those from the older patients $(7.7 \%)$ and at higher levels $[1.79 \pm 0.5$ and $0.5 \pm 0.2$, combined scores, in younger and older patients, respectively $(\mathrm{p}=0.027)]$. Among the $7 \mathrm{Msi}-1$-positive tumors in younger patients, 3 tumors developed from pyloric gland mucosa and 4 developed from fundic gland mucosa. Msi-1 expression was significantly associated with histologic type (poorly differentiated tubular adenocarcinoma, $\mathrm{p}=0.029$ ), invasion depth $(\mathrm{p}=0.036)$, lymph node metastasis $(\mathrm{p}=0.002)$ and overall stage $(\mathrm{p}=0.036)$ in the younger patients (Table II). However, no significant correlation was found 
Table II. Correlation of Msi-1 expression with clinicopathological features of young patients with gastric cancer.

\begin{tabular}{|c|c|c|c|c|}
\hline \multicolumn{5}{|c|}{ Age $\leq 30$ (mean age, 27.8$)$} \\
\hline Characteristics & $\begin{array}{c}\text { Msi-1(+) } \\
\mathrm{n}=7(\%)\end{array}$ & $\begin{array}{c}\text { Msi-1(-) } \\
\mathrm{n}=19(\%)\end{array}$ & $\begin{array}{c}\text { Total } \\
\mathrm{n}=26(\%)\end{array}$ & p-value \\
\hline Gender & & & & 0.353 \\
\hline Female & 3 (42.9) & $12(63.2)$ & $15(57.7)$ & \\
\hline Male & $4(57.1)$ & $7(36.8)$ & $11(42.3)$ & \\
\hline Histologic differentiation & & & & 0.029 \\
\hline Moderate & 2 (28.6) & $2(10.5)$ & $4(15.4)$ & \\
\hline Poor & $5 \quad(71.4)$ & $6(31.6)$ & $11(42.3)$ & \\
\hline Signet ring cell & $0 \quad(0.0)$ & $11(57.9)$ & $11(42.3)$ & \\
\hline T classification & & & & 0.036 \\
\hline $\mathrm{T} 1, \mathrm{~T} 2$ & $2(28.6)$ & $14(73.7)$ & $16(61.5)$ & \\
\hline $\mathrm{T} 3, \mathrm{~T} 4$ & $5 \quad(71.4)$ & $5(26.3)$ & $10(38.5)$ & \\
\hline Lymph node metastasis & & & & 0.002 \\
\hline No & 2 (28.6) & $17(89.5)$ & $19(73.1)$ & \\
\hline Yes & $5 \quad(71.4)$ & $2(10.5)$ & 7 (26.9) & \\
\hline Overall stage & & & & 0.036 \\
\hline I & 2 (28.6) & $14(73.7)$ & $16(61.5)$ & \\
\hline II, III & $5 \quad(71.4)$ & $5(26.3)$ & $10(38.5)$ & \\
\hline $\mathrm{Ki}-67$ & & & & 0.079 \\
\hline Negative & $1(14.3)$ & $10(52.6)$ & $11(42.3)$ & \\
\hline Positive & $6 \quad(85.7)$ & $9(47.4)$ & $15(57.7)$ & \\
\hline Post-operative metastasis & & & & 0.463 \\
\hline No & $5 \quad(71.4)$ & $16(84.2)$ & $21(80.7)$ & \\
\hline Yes & $2(28.6)$ & $3(15.8)$ & $5(19.3)$ & \\
\hline
\end{tabular}

between Msi-1 expression and clinicopathologic features in the older patients (Table III). Although Msi-1-positive carcinomas showed a trend toward higher cell proliferation as compared with Msi-1-negative carcinomas as revealed by Ki-67 immunostaining, this trend was not statistically significant $(\mathrm{p}=0.079)$. The level of Msi-1 expression was higher in the NCI-N87 and MKN-28 cell lines than in the MKN-45 and KATO III cell lines, as determined by western blot analysis (Fig. 2). This result confirmed the presence of Msi-1 protein in human gastric cancer.

Mucin phenotypes in Msi-1-positive gastric cancers. Results of immunostaining for mucin in Msi-1-positive gastric cancer are summarized in Table IV. In 9 Msi-1 positive gastric cancers, 6 were Muc5Ac-positive and 4 were Muc1-positive. The gastric foveolar type, observed in 5 of 9 tumors $(56 \%)$, was predominant, whereas no tumor of the gastric pyloric type was observed. Three of the 9 Msi-1 positive tumors $(33 \%)$ were of mixed gastrointestinal types.

Outcome. In 52 patients with gastric cancer (the 26 younger and 26 older patients matched for comparison), follow-up intervals ranged from 1.5 to 133.1 months (young patients; 1.5-133.1, older patients; 6.6-76.4 months). Eight patients died during the follow-up period. Mean survival (95\% confidence

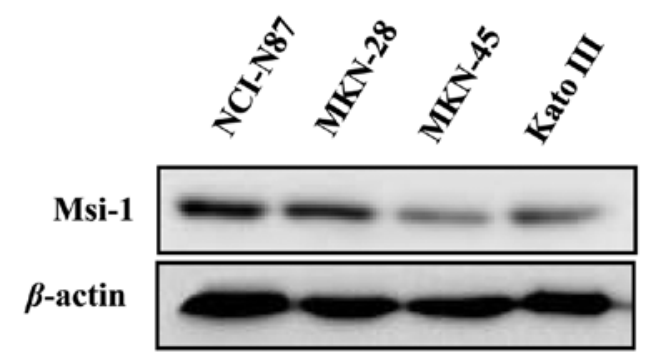

Figure 2. Western blot analysis confirmed Msi-1 protein expression in gastric cancer cell lines.

interval, CI) of the younger patients with gastric cancer was 123.6 months (95\% CI; 111.1-136.2). Mean survival of older patients was 63.9 months (95\% CI; 54.9-72.9). The 1-, 3- and 5 -year survival rates were 96,91 and $91 \%$ in young patients, and 81,76 and $76 \%$ in older patients, respectively. The two groups did not differ significantly in survival $(p=0.183)$. In the 26 young gastric cancer patients, Msi-1 expression was significantly associated with survival $(\mathrm{p}=0.014)$ in univariate Kaplan-Meier survival analysis. However, Msi-1 expression was not significantly associated with survival in the 26 older patients $(p=0.449)$ (Fig. 3). Multivariate survival analysis was not applied due to the small number of patients. 
Table III. Correlation of Msi-1 expression with clinicopathological features of elderly gastric cancer patients.

\begin{tabular}{|c|c|c|c|c|}
\hline \multicolumn{5}{|c|}{ Age $\geq 60$ (mean age, 68.8 ) } \\
\hline Characteristics & $\begin{array}{c}\text { Msi-1(+) } \\
n=2(\%)\end{array}$ & $\begin{array}{c}\text { Msi-1(-) } \\
n=24(\%)\end{array}$ & $\begin{array}{c}\text { Total } \\
\mathrm{n}=26(\%)\end{array}$ & p-value \\
\hline Gender & & & & 0.086 \\
\hline Female & $0 \quad(0.0)$ & $15(62.5)$ & $15(57.7)$ & \\
\hline Male & $2(100.0)$ & $9(37.5)$ & $11(42.3)$ & \\
\hline Histologic differentiation & & & & 0.821 \\
\hline Moderate & $0 \quad(0.0)$ & $4(16.6)$ & $4(15.4)$ & \\
\hline Poor & $1 \quad(50.0)$ & 10 (41.7) & $11(42.3)$ & \\
\hline Signet ring cell & $1 \quad(50.0)$ & $10(41.7)$ & $11(42.3)$ & \\
\hline T classification & & & & 0.284 \\
\hline $\mathrm{T} 1, \mathrm{~T} 2$ & $2(100.0)$ & $15(62.5)$ & $17(65.4)$ & \\
\hline $\mathrm{T} 3, \mathrm{~T} 4$ & $0 \quad(0.0)$ & $9(37.5)$ & $9(34.6)$ & \\
\hline Lymph node metastasis & & & & 0.326 \\
\hline No & $2(100.0)$ & $16(66.7)$ & $18(69.2)$ & \\
\hline Yes & $0 \quad(0.0)$ & $8(33.3)$ & $8(30.8)$ & \\
\hline Overall stage & & & & 0.245 \\
\hline I & $2(100.0)$ & $14(58.3)$ & $16(61.5)$ & \\
\hline II, III & $\begin{array}{ll}0 & (0.0)\end{array}$ & $10(41.7)$ & $10(38.5)$ & \\
\hline Ki-67 & & & & 0.819 \\
\hline Negative & $1 \quad(50.0)$ & $10(41.7)$ & $11(30.6)$ & \\
\hline Positive & $1 \quad(50.0)$ & $14(58.3)$ & $25(69.4)$ & \\
\hline Post-operative metastasis & & & & 0.595 \\
\hline No & $2(100.0)$ & $21(87.5)$ & $23(88.5)$ & \\
\hline Yes & $0 \quad(0.0)$ & $3(12.5)$ & $3(11.5)$ & \\
\hline
\end{tabular}

Table IV. Association between Msi-1 expression and mucin phenotype in gastric cancer.

\begin{tabular}{|c|c|c|c|c|c|c|c|c|}
\hline \multirow[b]{2}{*}{ No. } & \multirow[b]{2}{*}{ Age/gender } & \multirow[b]{2}{*}{ Histopathology } & \multicolumn{5}{|c|}{ Mucin expression (area $\mathrm{x}$ intensity) } & \multirow[b]{2}{*}{ Stage } \\
\hline & & & Muc1 & Muc2 & Muc5Ac & Muc6 & CD10 & \\
\hline 1 & $30 / \mathrm{M}$ & Adenocarcinoma, PD & $2 \times 3$ & 0 & $3 \times 3$ & 0 & 0 & pT3N2 \\
\hline 2 & $30 / F$ & Adenocarcinoma, PD & 0 & 0 & $1 \times 3$ & 0 & 0 & pT3N0 \\
\hline 3 & $24 / \mathrm{M}$ & Adenocarcinoma, MD & $1 \times 3$ & 0 & $2 \times 3$ & 0 & $1 \times 3$ & pT4bN3a \\
\hline 4 & $26 / \mathrm{F}$ & Adenocarcinoma, MD & 0 & 0 & $3 \times 3$ & 0 & 0 & pT1aN1 \\
\hline 5 & $23 / F$ & Adenocarcinoma, PD & $3 \times 3$ & 0 & 0 & 0 & 0 & pT3N3a \\
\hline 6 & 28/M & Adenocarcinoma, PD with SRCF & $1 \times 3$ & $2 \times 3$ & $2 \times 3$ & 0 & $2 \times 2$ & pT3N2 \\
\hline 7 & $29 / \mathrm{M}$ & Adenocarcinoma, PD with SRCF & 0 & $1 \times 3$ & $1 \times 3$ & 0 & 0 & pT1bN0 \\
\hline 8 & $73 / \mathrm{M}$ & Signet ring cell carcinoma & 0 & 0 & $2 \times 3$ & 0 & 0 & pT1aN0 \\
\hline 9 & $78 / \mathrm{M}$ & Adenocarcinoma, PD with SRCF & 0 & $1 \times 3$ & 0 & 0 & 0 & pT1bN0 \\
\hline
\end{tabular}

PD, poorly differentiated; MD, moderately differentiated; SRCF, signet ring cell foci.

\section{Discussion}

Gastric cancer is the fourth most common cancer and the second leading cause of cancer-related death $(1,2)$. Gastric cancer occurs predominantly in older age groups and gastric cancer patients younger than 30 years are very rare $(3-7,19)$.
Studies of gastric cancer in the young typically define patients 30-35 years or less as 'young' because intestinal metaplasia, an aging process affecting the gastric mucosa, is thought to begin at approximately this time of life (20). However, since actual number of patients younger than 30 years is very small, most studies of gastric cancer in young adults include patients 

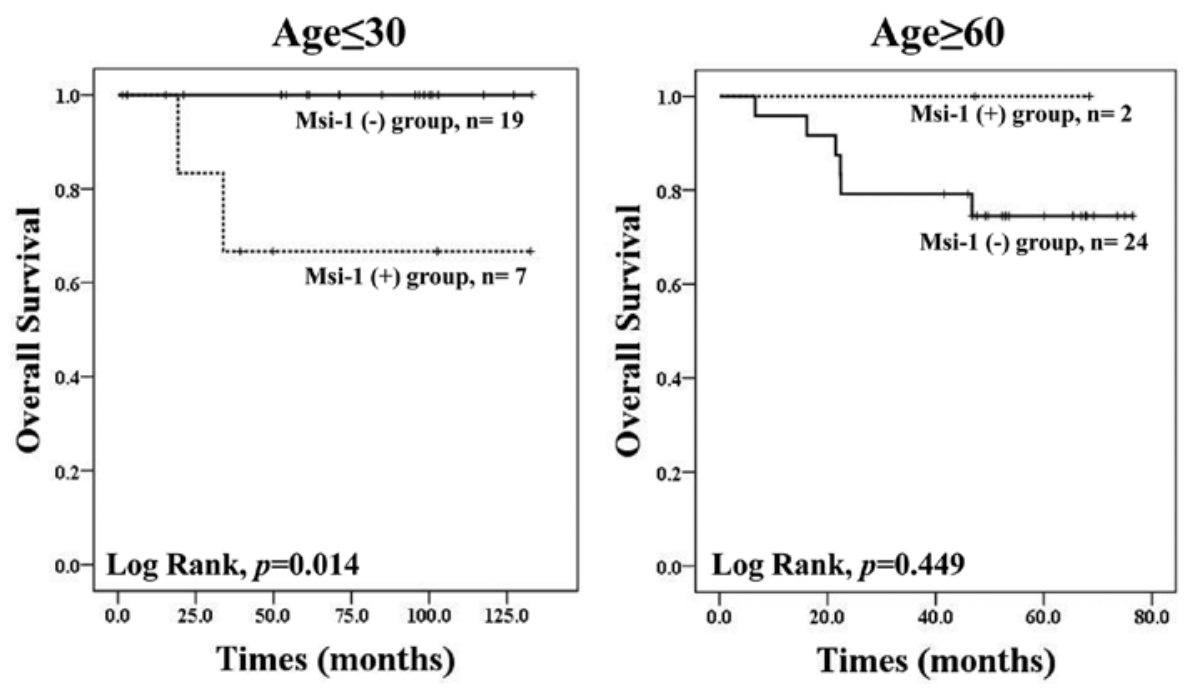

Figure 3. In 26 young gastric cancer patients, Msi-1 expression was significantly associated with patient survival ( $\mathrm{p}=0.014)$. In 26 older patients, Msi-1 expression was not significantly associated with survival. Log-rank $(\mathrm{p}=0.449)$.

up to 35 or 40 years (3-7). Although it has long been believed that gastric cancer in young patients shows a more aggressive phenotype and poorer prognosis than that in elderly patients, supportive data for such beliefs are uncertain (4-7). Because gastric cancer occurs most frequently in individuals 60 years of age or older, we used a case-control design to compare features of gastric cancer in the young patients represented in our database (age $\leq 30$ ) with those in an older group (age $\geq 60$ ) matched for important clinicopathological features, except age.

This study demonstrated for the first time the following characteristics of Musashi-1 expression in gastric cancer: i) more frequent detection and higher levels of expression in younger patients than in older ones; ii) significant associations with poorly differentiated histologic type, tumor invasion depth, lymph node metastasis, and overall stage in younger patients, but no significant correlations with clinicopathological features in older patients; iii) significant association with survival in younger patients; and iv) predominance of gastric foveolar mucin phenotype in Msi-1 positive gastric cancers. The expression of Msi-1 protein was confirmed directly in gastric cancer cell lines by western blot analysis. These findings support an active role for Msi-1 in development of gastric cancer in younger patients.

Msi-1 represents an evolutionarily conserved family of RNA-binding proteins with pivotal functions in stem cell maintenance, and nervous system development (10-13). Evidence that Msi-1 participates in carcinogenesis and tumor progression stems from studies of several human cancers (14,15,21-24). In gastric cancer, relationships between Msi-1 expression and clinicopathological features have not been fully examined. In this study, we found that Msi-1 expression correlated with high histologic grade, tumor invasion depth and lymph node metastasis of gastric cancer in young patients. Furthermore, a significant association was found between Msi-1 expression and overall survival in young patients. Our present findings in young patients with gastric cancer are consistent with studies of Msi-1 expression in other tumor types (23-25). In breast cancer patients, Msi-1 overexpression may be related to lymph node metastasis and shorter survival (23), and in human glioma, may be related to the degree of malignancy and proliferative activity (24). In patients with colon cancer, Msi-1 is associated with advanced cancer stage, metastasis and poor survival (25). Knockdown of Msi-1 with Msi-1 siRNA resulted in reduced colon cancer cell proliferation, cancer growth arrest in xenografts, and increased apoptosis alone and in combination with radiation therapy in athymic nude mice in vivo (14). In mouse intestinal cells, Msi-1 overexpression may induce tumors through Wnt and Notch activation (26). Our findings, therefore, together with previous studies support the contention that Msi-1 plays an important role in gastric cancer carcinogenesis and progression in young patients.

Our findings of significant associations in young gastric cancer patients with female gender and with diffuse type cancer, as compared to the 585 patients older than 31 years, are supported by previous studies showing higher proportions of female patients with poorly differentiated histology or diffuse type cancers in younger age groups (3-7). Our results, demonstrating the association of Msi-1 expression with poorly differentiated diffuse types of gastric cancer is in agreement with previous findings of significant increases in Msi-1 expression in diffuse gastric cancers as compared with the intestinal type of the cancer (27). In the present study, the expression of Msi-1 was also associated with gastric foveolar type gastric cancer showing Muc5Ac immunoreactivity. This is in agreement with previous studies demonstrating that Msi-1 expression overlapping with that of Muc5Ac, indicating that Msi-1 positive cells retain some features of foveolar cell differentiation (13). Previous studies also indicated that Muc5Ac expression is associated with a diffuse type of gastric cancer (28). Thus Msi-1 may be specifically involved in development of diffuse and foveolar types of gastric cancer. 
The level of Msi-1 expression was higher in the NCI-N87 and MKN-28 cell lines than in the MKN-45 and KATO III cell lines in this study. The NCI-N87 cells are derived from metastases of a well-differentiated gastric adenocarcinoma, and MKN-28 cells are derived from a moderately differentiated gastric adenocarcinoma that was non-metastatic. Conversely, MKN-45 cells are from a poorly differentiated gastric adenocarcinoma that was non-metastatic, and KATO III cells are signet ring cell carcinoma cells derived from pleural effusion. Therefore, the results obtained in vivo (i.e., higher Msi-1 expression in a poorly differentiated cell type) appear to contradict results obtained in vitro, where the highest level of Msi-1 expression was detected in NCI-N87 cells derived from a well-differentiated adenocarcinoma. The reasons for this discrepancy between in vivo and in vitro results are unclear. Among our young patients with gastric cancer, Msi-1 expression correlated significantly with a poorly differentiated cancer cell phenotype. However, the surgically resected specimens from 10 of 11 poorly differentiated carcinomas from elderly patients did not show positive Msi-1 expression. Moreover, MKN-28, MKN-45 and KATO III gastric cancer cell lines are derived from 70-, 62- and 55-year-old persons, respectively. The exact donor age was not reported for the NCI-N87 cell line. We conclude from these findings that Msi-1 expression may not always match the degree of cancer cell differentiation and potential for metastasis in gastric cancer, and that the relationship of Msi-1 expression to biological properties of the tumor may depend on the patient's age.

The relationship between patient age and prognosis in gastric cancer remains controversial. Gastric cancer in young patients has long been believed to show more aggressive biologic behavior and poorer prognosis than that in elderly patients (4-7). In our case-control study with matched subjects, the survival rates for young patients were better than those for older patients, although the difference between survival rates was not significant. These findings are in agreement with a number of prior reports showing similar survival rates in young and elderly patients $(3,20,29)$. Moreover, Eguchi et al reported better prognosis in young patients with gastric cancer and noted that early diagnosis improves the prognosis (19). Taken together, our data suggested that though young patients may present with advanced stages of cancer and tumors of poorly differentiated histology, age itself may not independently predict survival outcome for young patients. Based on the limited number of our cases and insufficient follow-up periods, a definitive conclusion on the prognosis of young gastric cancer patients would be premature. A longerterm follow-up with a larger cohort is needed to adequately define the clinical and biological behavior of gastric cancer in young patients.

In conclusion, our study revealed significantly higher tumor Msi-1 expression in young gastric cancer patients than in elderly patients, and an association of Msi-1 expression with aggressive gastric cancer behavior in young patients.

\section{Acknowledgements}

This study was supported by a grant from the National Research Foundation of Korea (NRF) funded by the Korean Government
(MSIP) (No. 2008-0062279). Tissue samples were provided by the Chonbuk National University Hospital, a member of the National Biobank of Korea, which is supported by the Ministry of Health, Welfare and Family Affairs.

\section{References}

1. Jemal A, Bray F, Center MM, Ferlay J, Ward E and Forman D: Global cancer statistics. CA Cancer J Clin 61: 69-90, 2011.

2. Leung WK, Wu MS, Kakugawa Y, et al: Screening for gastric cancer in Asia: current evidence and practice. Lancet Oncol 9: 279-287, 2008.

3. Bai Y and Li ZS: Endoscopic, clinicopathological features and prognosis of very young patients with gastric cancer. J Gastroenterol Hepatol 26: 1626-1629, 2011.

4. Nakamura R, Saikawa Y, Takahashi T, et al: Retrospective analysis of prognostic outcome of gastric cancer in young patients. Int J Clin Oncol 16: 328-334, 2011.

5. Theuer CP, de Virgilio C, Keese G, et al: Gastric adenocarcinoma in patients 40 years of age or younger. Am J Surg 172: 473-476, 1996.

6. Quijano Orvananos F, Moreno Paquentin E, Alvarez JJ, Martinez Munive A and Butron Perez L: Gastric carcinoma in patients under 35 years. Rev Gastroenterol Mex 64: 75-77, 1999.

7. Smith BR and Stabile BE: Extreme aggressiveness and lethality of gastric adenocarcinoma in the very young. Arch Surg 144: 506-551, 2009.

8. Saikawa Y, Fukuda K, Takahashi T, Nakamura R, Takeuchi H and Kitagawa Y: Gastric carcinogenesis and the cancer stem cell hypothesis. Gastric Cancer 13: 11-24, 2010.

9. Clarke MF, Dick JE, Dirks PB, et al: Cancer stem cells perspectives on current status and future directions: AACR Workshop on cancer stem cells. Cancer Res 66: 9339-9344, 2006.

10. Visvader JE and Lindeman GJ: Cancer stem cells in solid tumours: accumulating evidence and unresolved questions. Nat Rev Cancer 8: 755-768, 2008.

11. Nakamura M, Okano H, Blendy JA and Montell C: Musashi, a neural RNA-binding protein required for Drosophila adult external sensory organ development. Neuron 13: 67-81, 1994.

12. Potten CS, Booth C, Tudor GL, et al: Identification of a putative intestinal stem cell and early lineage marker; musashi-1. Differentiation 71: 28-41, 2003.

13. Akasaka Y, Saikawa Y, Fujita K, et al: Expression of a candidate marker for progenitor cells, Musashi-1, in the proliferative regions of human antrum and its decreased expression in intestinal metaplasia. Histopathology 47: 348-356, 2005.

14. Sureban SM, May R, George RJ, et al: Knockdown of RNA binding protein musashi-1 leads to tumor regression in vivo. Gastroenterology 134: 1448-1458, 2008.

15. De Sousa Abreu R, Sanchez-Diaz PC, Vogel C, et al: Genomic analyses of musashil downstream targets show a strong association with cancer-related processes. J Biol Chem 284: 12125-12135, 2009.

16. Edge SB, Byrd DR, Compton CC, Fritz AG, Greene FL and Trotti A: AJCC Cancer Staging Manual. 7th edition. Springer, New York, 2010.

17. Lauwers GY, Carneiro F, Graham DY, et al: Gastric carcinoma. In: WHO Classification of Tumours of the Digestive System. 4th edition. IARC Press, Lyon, p48-58, 2010.

18. Lauren P: The two histological main types of gastric carcinoma, undifferentiated and so-called differentiated-type carcinoma. Acta Pathol Microbiol Scand 64: 31-49, 1965.

19. Eguchi T, Takahashi Y, Yamagata M, Kasahara M and Fujii M: Gastric cancer in young patients. J Am Coll Surg 188: 22-26, 1999.

20. Katai H, Sasako M, Sano T and Maruyama K: Gastric carcinoma in young adults. Jpn J Clin Oncol 26: 139-143, 1996.

21. Shu HJ, Saito T, Watanabe H, et al: Expression of the Musashil gene encoding the RNA-binding protein in human hepatoma cell lines. Biochem Biophys Res Commun 293: 150-154, 2002.

22. Wang T, Ong CW, Shi J, et al: Sequential expression of putative stem cell markers in gastric carcinogenesis. Br J Cancer 105: 658-665, 2011. 
23. Wang XY, Penalva LO, Yuan $\mathrm{H}$, et al: Musashi1 regulates breast tumor cell proliferation and is a prognostic indicator of poor survival. Mol Cancer 9: 221, 2010.

24. Toda M, Iizuka Y, Yu W, et al: Expression of the neural RNA-binding protein Musashil in human gliomas. Glia 34: 1-7, 2001.

25. Li D, Peng X, Yan D, et al: Msi-1 is a predictor of survival and a novel therapeutic target in colon cancer. Ann Surg Oncol 18 : 2074-2083, 2011.

26. Rezza A, Skah S, Roche C, Nadjar J, Samarut J and Plateroti M: The overexpression of the putative gut stem cell marker Musashi-1 induces tumorigenesis through Wnt and Notch activation. J Cell Sci 123: 3256-3265, 2010.
27. Nikpour P, Emadi-Baygi M, Mohhamad-Hashem F, Maracy MR and Haghjooy-Javanmard S: MSI1 overexpression in diffuse type of gastric cancer. Pathol Res Pract 209: 10-13, 2013

28. Pinto-de-Sousa J, David L, Reis CA, Gomes R, Silva L and Pimenta A: Mucins MUC1, MUC2, MUC5AC and MUC6 expression in the evaluation of differentiation and clinicobiological behaviour of gastric carcinoma. Virchows Arch 440: 304-310, 2002.

29. Kim DY, Joo JK, Ryu SY, Park YK, Kim YJ and Kim SK: Clinicopathologic characteristics of gastric carcinoma in elderly patients: a comparison with young patients. World J Gastroenterol 11: 22-26, 2005. 